SCIENTIFIC LETTER

\title{
A population based study of Ramadan fasting and acute coronary syndromes
}

\author{
J Al Suwaidi, A Bener, A Suliman, R Hajar, A M Salam, M T Numan, H A Al Binali
}

Heart 2004;90:695-696. doi: 10.1136/hrt.2003.012526

$\mathrm{S}$ ustained fasting over a period is a feature of several of the world's great religions. One of the five fundamental rituals of Islam is fasting during the month of Ramadan. Muslims neither eat nor drink anything from dawn until sunset. Fasting may have negative effects on cardiac patients because of the limited time allowed for food intake and the heavy physical worship that is performed after a heavy meal, as well as the inability to take any medications during fasting, which may be essential for the patients. ${ }^{1}$

Despite the fact that most clinicians worldwide treat Muslim patients, data on the incidence of patients presenting with acute coronary syndromes (ACS) in relation to fasting during Ramadan are lacking. Hence, the purpose of this study is to investigate whether Ramadan fasting has a negative effect on the incidence of presentation with ACS such as acute myocardial infarction (AMI) and unstable angina (UA).

\section{METHODS}

This study was based at Hamad General Hospital, Doha, Qatar, which is the only tertiary care centre in the country and so all patients with ACS are treated here. The Hamad General Hospital cardiology database was used for this study; this database comprises data collected from all patients admitted to the cardiology department at the hospital since January 1991. Data were collected from the clinical records by the patients' physicians at the time of the patients' hospital discharge according to predefined criteria for each data point. We focused our study only on Qatari patients because it is a stable population and avoids the bias in the fluctuation of the expatriate population in the country. Furthermore, more than 95\% of Qatari adults regularly practise fasting. The study was approved by the institution review board.

With the described database, all patients presenting with ACS from the year 1991 to 2001 were identified. Age, sex, risk factors including smoking, hypertension, hypercholesterolaemia, diabetes, pre-existing coronary heart disease, in-hospital mortality and morbidity, as well as acute medical care provided were analysed.

Periods corresponding to the month of Ramadan in the Gregorian calendar have been established, since the lunar calendar is 11-12 days shorter than the solar year. To reduce the effects originating from seasonal changes to a minimum and to use the data as a control, we evaluated patients for one month before, during, and one month after Ramadan by going back and forward from the period of Ramadan (29-30 days) in a particular year.

Data are expressed as mean and standard deviation of the mean. One way analysis of variance (ANOVA) $t$ test, $\chi^{2}$ test, and Fisher's exact test were used for statistical analysis. A probability value of $\mathrm{p}<0.05$ was considered significant.

\section{RESULTS}

During the 10 year period, a total of 20856 patients were admitted to the coronary care unit and cardiology wards;
8446 of them were Qataris and 12410 were non-Qataris. There was no significant difference among the three periods (one month before, during, and after Ramadan) in regards to the incidence of AMI (123, 142, and 150 patients, respectively, $\mathrm{p}>0.05)$. Furthermore, the clinical characteristics of these patients such as age (61 (12) years, 62 (14) years, and 60 (13) years), sex (male $76.5 \%, 72.5 \%$, and $73 \%$ ), smoking status $(23 \%, 20 \%$, and $26 \%)$, presence of hypertension (33.5\%, 39\%, and 38\%), hypercholesterolaemia (23.5\%, 25\%, and $25 \%)$, diabetes $(58 \%, 51 \%$, and $53 \%)$, prior AMI $(15 \%$, $19 \%$, and $19 \%)$, and prior coronary artery bypass grafting $(3.2 \%, 3.5 \%$, and $3 \%)$ were not significantly different. Patients who were admitted after Ramadan were more likely to smoke more packets per day of cigarettes when compared to the other two time periods $(0.84,0.91$, and 1.32 packets, respectively, $\mathrm{p}<0.001$ ).

No significant differences were found among patients admitted with UA in the three periods (160, 146, and 147 patients, respectively, $p>0.05)$. Clinical characteristics of these patients such as age (60 (12) years, 60 (12) years, and 59 (12) years), sex (male 58.8\%, 50.0\%, and 60\%), smoking $(17.8 \%, 9.6 \%$, and $19.2 \%)$, presence of hypertension $(49 \%$, $54 \%$, and $48 \%)$, hypercholesterolaemia $(27 \%, 27 \%$, and $23 \%)$, diabetes $(51 \%, 56 \%$, and $59 \%)$, and pre-existing cardiac disease were also not significantly different. Statistical analysis showed no significant differences in thrombolysis administration $(27 \%, 25 \%$, and $27 \%)$, death $(9 \%, 10.4 \%$, and $10.7 \%)$, bleeding $(0.3 \%, 0 \%$, and $0.3 \%)$ or stroke $(0.3 \%, 0.7 \%$, and $1 \%$ ) among patients in the three periods.

\section{DISCUSSION}

In this population based study, we found no significant differences in the incidence of AMI or UA during Ramadan when compared to the rest of the year.

Previous studies documented an association between Ramadan fasting and biochemical and hormonal changes. Several investigators reported changes in lipid profile; however, these results were based on a small number of patients and were contradictory. ${ }^{1}$ Fasting has been associated with variations in the incidence of some diseases, however, the incidence of stroke in Ramadan was not significantly different from the rest of the year. ${ }^{2}$

Despite the fact that fasting during Ramadan is practised by more than a billion Muslim people worldwide, data on the incidence of cardiac diseases are sparse. There are only two reported studies on the incidence of ACS during Ramadan. ${ }^{3}$ Gumaa and colleagues, ${ }^{4}$ reported an increase in complaints of angina during Ramadan. More recently, Temizhan and colleagues $^{3}$ reported no significant differences in the incidence of ACS during Ramadan when compared to one month before and after Ramadan in 1655 patients. However, this

Abbreviations: ACS, acute coronary syndromes; $A M I$, acute myocardial infarction; UA, unstable angina 
study had many limitations including the fact that it was not population based, only included a small number of patients, and did not define the exact number of patients with ACS who were actually fasting. The last point is particularly important, considering the patients were from a community where more than $35 \%$ of the population do not regularly fast during Ramadan. ${ }^{4}$ The current study extends the findings of Temizhan and colleagues ${ }^{3}$ in a more defined population where more than $95 \%$ of the population regularly fast. Furthermore, it describes for the first time complete clinical characteristics, mode of treatment, and outcome of patients with ACS. In conclusion, we speculate that Ramadan fasting does not increase ACS.

\section{Authors' affiliations}

J Al Suwaidi, A Suliman, R Hajar, A M Salam, M T Numan, H A Al Binali, Department of Cardiology and Cardiovascular Surgery, Hamad General Hospital and Hamad Medical Corporation, Doha, State of Qatar
A Bener, Department of Biostatistics and Epidemiology, Hamad General Hospital and Hamad Medical Corporation

Correspondence to: J Al Suwaidi, Department of Cardiology and Cardiovascular Surgery, Hamad Medical Corporation and Hamad General Hospital, PO Box 3050, Doha, State of Qatar; jha01@hmc.org.qa

Accepted 25 September 2003

\section{REFERENCES}

1 Azizi F. Research in Islamic fasting and health. Ann Saudi Med 2002;22:186-191.

2 Akhan G, Kutluhan S, Koyuncuoglu $\mathrm{HH}$. Is there any change of stroke incidence during Ramadan? Acta neurol scan 2000;101:259-61.

3 Temizhan A, Donderici O, Ouz D, et al. Is there any effect of Ramadan fasting on acute coronary heart disease events? Int J Cardiol 1999:70:149-53.

4 Gumaa KA, Mustafa KY, Mahmoud NA, et al. The effect of fasting in Ramadan: Serum uric acid and lipid concentration. $\mathrm{Br} J$ Nutr 1978:40:573-81.

\section{IMAGES IN CARDIOLOGY}

doi: $10.1136 /$ hrt.2003.027086

\section{Right ventricular ischaemia due to right coronary artery stenosis}

A 47 year old man, without a history of hypertension, was admitted for chest pain. Stress and rest 201 -thallium perfusion scintigraphy was performed, using dipyridamole and bicycle exercise $(50 \mathrm{~W}$ workload). The stress image (panel A) revealed diminished tracer uptake in the inferior wall of the left ventricle (LV) and the inferoseptal wall of the right ventricle (RV). On the rest image (panel
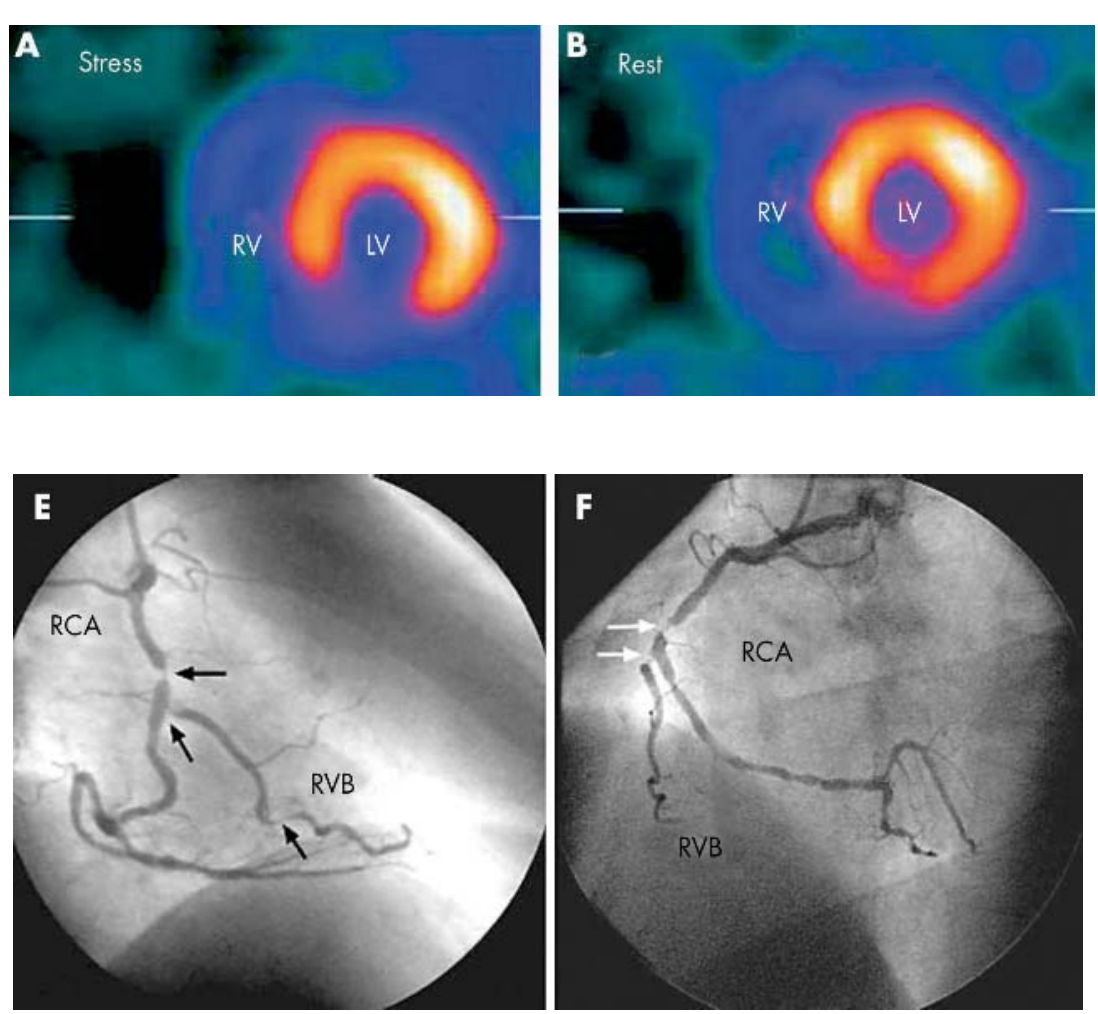

B), acquired four hours later, almost complete redistribution was demonstrated which indicates both LV and RV ischaemia. In addition, the stress ECG (panel C) showed ST segment depression in the inferior leads and ST segment elevation in leads aVR and $\mathrm{V}_{\mathrm{l}}$, reflecting both $\mathrm{LV}$ and $\mathrm{RV}$ ischaemia. Compared to the rest ECG (panel D) P wave amplitude increased during stress, which may have been caused by
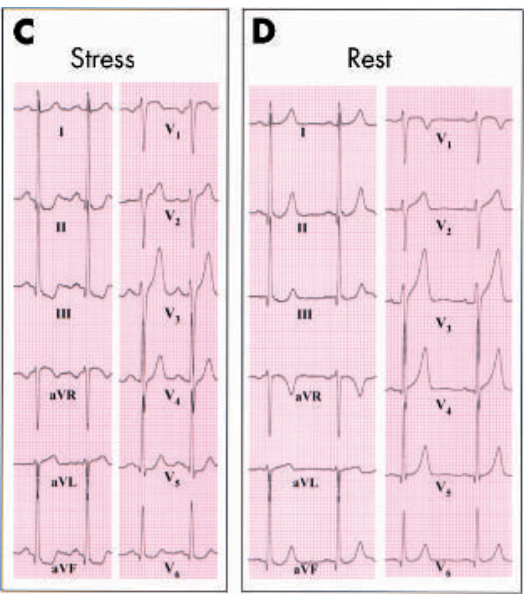

increased atrial pressure as a result of transient end diastolic pressure elevation caused by stress induced myocardial ischaemia. Subsequent coronary angiography (panels $\mathrm{E}$ and $\mathrm{F}$, right anterior oblique and left anterior oblique projection, respectively) demonstrated significant stenosis of the right coronary artery (RCA) proximal to the take off of the right ventricular branch (RVB) which shows an ostial and distal stenosis (see arrows). Coronary angiography showed no stenosis in the left coronary artery.

This case demonstrates that stress/ rest 201 thallium perfusion scintigraphy and stress ECG enables the detection of $\mathrm{RV}$ ischaemia caused by significant RCA stenosis.

G A Somsen, E Camenzind, A Righetti aernout.somsen@hcuge.ch 